Volume: 2, Issue: 1, January-March 2017, Pages: 130, DOI: http://dx.doi.org/10.19082/ah130

\title{
DESIGN OF CAD SYSTEM OF SOLITARY PULMONARY NODULE WITH HARMONY CLASSIFICATION AND FUZZY SYSTEM
}

\author{
Maryam Sadat Mahmoodi ${ }^{1, *}$, Seyed Abbas Mahmoodi ${ }^{2}$
}

1: Department of Computer Endineering, Payame Noor University (PNU), Tehran, Iran.

2: Department of Computer Engineering, Yazd Science and Research Branch, Islamic Azad University, Yazd, Iran

\section{TYPE OF ARTICLE: CONFERENCE ABSTRACT}

\begin{abstract}
Introduction: Lung cancer is the most widespread form of cancer, with the highest mortality rate worldwide. In this study, a computer-aided detection (CAD) system was developed for lung nodule detection, segmentation, and recognition using CT images. Thus, we use a highly accurate supervised test that includes lung images with the aim of assisting physicians in early detection of lung cancer.

Methods: First, we segmented the lung area by masking techniques to isolated nodules and determined region of interest. Then, 24 features were extracted from images that included morphological, statistical, and histogram. Important features were derived from the images for their posterior analysis with the aid of a harmony search algorithm and fuzzy systems.

Results: In order to evaluate the performance of the proposed method, we used the LIDC database, the number of images included a database of 97 images from 47 patients diagnosed with lung cancer. Results of the base method show a sensitivity of $93 \%$.

Conclusion: The harmony search algorithm is optimized using a fuzzy system for classification. The CAD system provides $93.1 \%$ accuracy.

KEYWORDS: Lung cancer, Solitary pulmonary nodule, CT image, Harmony algorithm, Fuzzy system
\end{abstract}

\footnotetext{
Abstracts of First National Congress of Medical Informatics, Mashhad, Iran, February 2017

(C) 2017 The Authors. This is an open access article under the terms of the Creative Commons Attribution-NonCommercialNoDerivs License, which permits use and distribution in any medium, provided the original work is properly cited, the use is non-commercial and no modifications or adaptations are made.
} 